\title{
Efficacy of Leukotriene Modifiers for the Treatment of Persistent Asthma in Children
}

\author{
Duarte G. Machado*
}

\begin{abstract}
The purpose of this study was to evaluate the use of the leukotriene modifiers (LTMs), zafirlukast and montelukast, in children with asthma managed by an inner city pediatric pulmonary practice. A retrospective chart review was done of children 6 years of age with persistent asthma seen at Connecticut Children's Medical Center and prescribed LTM drugs. Eighty-three children whose asthma control was adequately assessed both before and after addition of a LTM to his/her treatment regimen was included in the study. There were statistically significant improvements in several parameters of asthma control following initiation of LTM use, including provider assessment score $(p=\mathbf{0 . 0 0 0 5})$, number of hospitalizations and unscheduled visits (clinic or emergency department; $p<0.0001)$, use of oral corticosteroids $(p=0.0015)$, spirometry severity score $(p=0.0015)$, and spirometry test results (FEV1, FEV1/FVC, FEF, FEF25-75\%; $p<0.005$ for all). These results suggest that montelukast and zafirlukast help to improve asthma control in young patients with persistent asthma.
\end{abstract}

\section{INTRODUCTION}

Asthma is the most common chronic illness of childhood in the United States, affecting an estimated 4.8 million children, or $10 \%$ of the pediatric population (1). In 1997, the National Heart, Lung and Blood Institute (NHLBI) issued a report of recommended guidelines for the diagnosis and management of asthma, which included the use of inhaled corticosteroids (ICS) as the primary controller for persistent disease (2). Asthma is classified as persistent when symptoms occur $>2$ times a week and nighttime symptoms occur $>2$ times a month (2). According to the above guidelines, the standard therapeutic regimes for children with mildly persistent asthma include a daily antiinflammatory (either ICS, cromolyn or nedocromil) or sustained-release theophylline, while a short-acting bronchodilator, such as an inhaled beta2-agonist, is used

To whom correspondence should be addressed:

* Duarte G. Machado, University of Connecticut School of Medicine, 263 Farmington Ave., Farmington, CT 06030 and Research Assistant, Department of Pulmonary Medicine, Connecticut Children's Medical Center, 282 Washington St., Hartford, CT 06106 for quick relief of acute attacks.

However, the use of ICS in pediatric asthma raises several concerns. ICS can adversely affect several systems of the body and lead to adrenal axis suppression and growth inhibition (3). Prepubertal children are at greatest risk for experiencing growth suppression induced by ICS (4). In addition, ICS may be complicated to administer because proper metered-dose inhaler technique, spacers, and multiple doses during the day may be required (5). Other controller medications for persistent asthma also have undesirable characteristics. Patients who use beta2-agonists may experience anxiety, tremor, restlessness, irritability, insomnia, tachycardia, elevated blood pressure, paradoxical bronchospasm, and hypokalemia (6). Moreover, theophylline has a narrow therapeutic range and its use in children has been associated with restlessness, agitation, diuresis, and fever (7). Since rates of theophylline absorption and metabolism vary widely among patients and in the same patient at different times, regular monitoring of plasma drug concentration is necessary for optimal control (7). 
Table 1. Comparison of Leukotriene Modifiers $(11,13,16,17,18)$

\begin{tabular}{|c|c|c|c|}
\hline Name & zafirlukast (Accolate) & zileuton (Zyflo) & montelukast (Singulair) \\
\hline Mechanism of action & $\begin{array}{l}\text { selective and competitive cys-LT1 } \\
\text { receptor antagonist }\end{array}$ & $\begin{array}{l}\text { selectively and reversibly } \\
\text { inhibits the 5-lipoxygenase } \\
\text { pathway }\end{array}$ & $\begin{array}{l}\text { selectively blocks the cys-LT1 } \\
\text { receptor }\end{array}$ \\
\hline Age Indication & $>/=5$ years & $>/=12$ year & $>/=2$ years \\
\hline Usual dose & $\begin{array}{l}20 \mathrm{mg} \text { tablet bid ( } 12 \text { yrs of age); } \\
10 \mathrm{mg} \text { tablet bid non-flavored mini } \\
\text { tablet ( } 5-11 \text { yr old children), }\end{array}$ & $\begin{array}{l}600 \mathrm{mg} \text { tablet qid } \\
(12 \mathrm{yrs} \text { of age })\end{array}$ & $\begin{array}{l}10 \mathrm{mg} \text { tablet } \mathrm{Qhs}(15 \mathrm{yrs} \text { of } \\
\text { age) } 5 \mathrm{mg} \text { chewable tablets } \\
(6-14 \mathrm{yr} \text { children }) ; 4 \mathrm{mg} \text { cherry- } \\
\text { flavored chewable tablets ( } 2-5 \\
\text { yr old children) }\end{array}$ \\
\hline Warnings & ? Churg-Strauss syndrome? & $\begin{array}{l}\text { elevated LFTs, monitor } \\
\text { LFTs at initiation and } \\
\text { during therapy }\end{array}$ & ? Churg-Strauss syndrome? \\
\hline Dosing considerations & $\begin{array}{l}\text { empty stomach ( } 1 \mathrm{hr} \text { before or } 2 \mathrm{hrs} \\
\text { after); food decreases absorption } \\
\text { by } 40 \%\end{array}$ & none & none \\
\hline Drug interactions & $\begin{array}{l}\text { warfarin (increase PT), phenytoin, } \\
\text { carbamazepine }\end{array}$ & $\begin{array}{l}\text { warfarin (increase PT) } \\
\text { theophylline (increase) } \\
\text { propranolol (increase) }\end{array}$ & none \\
\hline
\end{tabular}

Abbreviations: cys-LT1, cysteinyl leukotriene; LFT, liver function test; PT, prothrombin time

The newest class of drugs, leukotriene modifiers, may be an effective alternative, especially for children. Produced and released from eosinophils, mast cells, and alveolar macrophages, cysteinyl leukotrienes are thought to play a direct role in the pathogenesis of asthma. Through activation of at least two seven transmembrane-spanning receptors, CysLT1

and CysLT2, leukotrienes can stimulate mucus formation and secretion, edema, and contraction and proliferation of smooth muscle cells (8). These effects are associated with cellular infiltration of the airways and decreased mucociliary transport. Cysteinyl leukotrienes are also classified as strong bronchoconstrictors as they are up to 1,000 times more potent than histamine (9). There is now sufficient evidence to suggest that drugs that target and modify the leukotriene pathway have the potential to alter the pathogenesis of asthma (10).

To date, three chemically distinct LTMs are available by prescription. Zafirlukast (Accolate ; Astra Zeneca Pharmaceuticals, Wilmington, DE) was approved in September 1996, followed by zileuton (Zyflo ; Abbott Laboratories, Chicago, IL) in January 1997, and montelukast (Singulair ; Merck \& Co., Inc. Whitehouse Station, NJ) in February 1998 (11-13). LTMs comprise two pharmacologic classes of compounds. Zileuton inhibits the enzyme 5-lipoxygenase, thereby preventing the biosynthesis of leukotrienes. In contrast, zafirlukast and montelukast are CysLT1 selective receptor antagonists that block the binding of leukotrienes to this receptor type (14). Importantly, these antagonists selectively block binding to only the CysLT1 receptor, which is highly expressed in lung smooth muscle cells and interstitial lung macrophages (as well as the spleen and peripheral blood leukocytes including eosinophils) (15). In contrast, the CysLT2 receptor is not expressed in the lung at all, but instead in the heart, adrenal medulla, placenta, and peripheral blood leukocytes (15). Important features of the three drugs are given in Table 1 .

The clinical trials that led to the FDA approvals of the different LTMs had all demonstrated improvements in several parameters of asthma control to some degree. In a study of asthmatic adults, it was found that patients treated with zafirlukast experienced a significant decrease in nocturnal symptoms, a significant improvement in forced expiratory volume in one second (FEV1), and a $30 \%$ or greater improvement of asthma symptoms compared to those given placebo (19). Improvement in asthma symptoms was found to occur within hours after administration of zafirlukast (11). In these studies, zafirlukast was used alone or in combination with rescue medication as needed. Approval for use in patients 12 years of age was granted 
two years later based on these studies. Studies of zafirlukast use in children aged five to 11 years were subsequently performed to assess whether or not similar results as found in adult patients could be achieved. A four-week, double blind trial and 52 week open-label extension of children with mild to moderate asthma concluded that zafirlukast was generally well-tolerated and effective (20). A $10 \mathrm{mg}$ pediatric formulation of zafirlukast was subsequently granted approval for use in patients 5 years of age.

Use of montelukast in 6 to 14-year-old children was first addressed in an eight-week, double blind trial completed in April 1996. It was found that montelukast significantly decreased daily as-needed use of agonists, the mean percentage of days with an asthma exacerbation, and the percentage of patients who experienced at least one asthma exacerbation (5). Montelukast also allowed for significant tapering of inhaled corticosteroids. This study provided sufficient evidence to allow for the approval of montelukast two years later for use in children 6 years of age. A $4 \mathrm{mg}$ tablet was approved for patients 2 years of age in March 2000 following extrapolation of the efficacy of montelukast (13). Clinical studies have now been extended to patients $<6$ years of age, for whom montelukast was found to significantly protect against bronchoconstriction following a cold, dry air challenge (21). Moreover, both zafirlukast and montelukast have been demonstrated to provide modest but statistically significant improvement in lung function when used as monotherapy in children as young as 6 years of age $(5,22,23)$.

Although LTMs have been shown to be safe and effective in clinical trials, the optimum use of LTMs in asthma management is still evolving (24). Since LTMs had only become commercially available shortly before the 1997 NHLBI guidelines were drafted, the guidelines were based on limited published data examining their use in asthma treatment (25). Moreover, there are still inadequate data regarding the efficacy of regular use of LTMs in children with chronic asthma (5). The current study was undertaken to evaluate the use of LTMs in the treatment of children with asthma managed by a pediatric pulmonary practice in Connecticut. Our hypothesis was that addition of LTM therapy would result in improved control of symptoms and lung function in children with chronic persistent asthma.

\section{MATERIALS AND METHODS Study Design}

All patients greater than or equal to 6 years of age with persistent asthma who had at least two asthmarelated clinical encounters with the pediatric pulmonary practice at Connecticut Children's Medical Center (CCMC) from September 1996 to March 2000 were eligible for inclusion in this study and were identified using a computerized database. The cutoff date was selected as the date when zafirlukast gained FDA approval for use in children. Since zileuton was not used in this practice, all patients were prescribed either montelukast or zafirlukast.

The medical records of 279 children with asthma at CCMC were specifically reviewed for LTM use. Pre and post clinical information was then compiled from their charts. Pre was defined as the first encounter in which a clinical assessment, which included exercise tolerance, spirometry and other pulmonary function tests performed by a pediatric pulmonology technician, concluded with a prescription for a LTM. Since the effects of LTMs were previously noted to occur as early as within one day after the first dose (21), post was defined as the first clinical encounter since initiation of LTM therapy.

\section{Subjects}

Of the 279 children, 113 met the initial inclusion criterion of having used a LTM. Of these, 30 children were excluded from the study for one or more of the following reasons: (a) the majority of the pre and post-treatment information was not available, (b) they were already taking LTMs at the first clinical encounter via prescription from another provider, or (c) they had failed to keep one or more follow-up appointments at the clinic such that post-treatment asthma control could not be ascertained. The final sample size was therefore 83 children.

The 83 patients with complete data for review had a mean age of $10.6 \pm 0.3$ years (ranging from 5 to 16 years). More subjects were in the montelukast group (72, or $86.7 \%$ ) compared to the zafirlukast group (11, or $13.3 \%)$. The fewer subjects in the latter group reflected an early concern in using zafirlukast because it is also an inhibitor of the CYP450 isoenzyme CYP3A4, such that zafirlukast can increase concentrations of certain concomitant medications, including theophylline (26). In addition, the greater number of children in the montelukast group was not biased by the 30 children who were excluded since most of these were also prescribed montelukast (26, or $86.7 \%$ ) rather than zafirlukast (4, or $13.3 \%)$.

Asthma severity was classified as severe persistent for 42 patients $(50.6 \%)$, moderate persistent for 32 $(38.6 \%)$, and mild persistent for 9 (10.8\%), respectively. Sixty (87\%) subjects reported allergies or were skin-tested positive for one or more allergens. Moreover, sixty (74\%) subjects, not necessarily the same ones, also had a diagnosis of allergic rhinitis. At 
Table 2. Pre and Post Assessment Score Ratings

\begin{tabular}{|c|c|c|c|c|}
\hline Name & $\mathbf{0}$ & 1 & 2 & 3 \\
\hline Frequency of Nocturnal awakenings & none & $<1 /$ week & $>=1 /$ week & \\
\hline Exercise Tolerance & good & limited & severly limited & \\
\hline School absenteeism & none & $<=10 /$ year & $>10 /$ year & \\
\hline $\begin{array}{l}\text { Provider's assessment of asthma } \\
\text { control }\end{array}$ & satisfactory & marginal & unsatisfactory & \\
\hline Rescue use of short acting bronchodilators & none & $<=1 /$ week & $>1 /$ week & \\
\hline Number of hospitalizations or sick visits & none & 1/year & 2/year & $>=3 /$ year \\
\hline Use of oral steroids in past six months & no & yes & & \\
\hline Spirometry Severity & normal & mild & moderate & severe \\
\hline
\end{tabular}

Table 3. Patient Demographics

\begin{tabular}{lcccc}
\hline & Overall & Male & Female & $\begin{array}{c}\text { Level of } \\
\text { Significance }\end{array}$ \\
\hline Number & 83 & 40 & 43 & \\
Age (yrs) & $10.6+/-0.3$ & $10.3+/-0.3$ & $10.9+/-0.3$ & \\
$\begin{array}{l}\text { Allergies } \\
\text { (n=69)* }\end{array}$ & $60(87 \%)$ & $33(94 \%)$ & $27(79 \%)$ & \\
$\begin{array}{l}\text { Allergic } \\
\text { rhinitis } \\
\text { (n=81)* }\end{array}$ & $60(74 \%)$ & $27(69 \%)$ & $33(79 \%)$ & \\
$\begin{array}{l}\text { Spirometry } \\
\text { severity }\end{array}$ & & $1.4+/-0.12$ & $0.9+/-0.11$ & 0.0014 \\
score & & & & \\
$\begin{array}{l}\text { FEV1/FVC } \\
\text { ratio }\end{array}$ & & $76.6+/-1.4$ & $82.7+/-1.0$ & 0.0005 \\
\begin{tabular}{l} 
FEF (25-75\%) \\
\hline
\end{tabular} & $62.2+/-2.9$ & $80.8+/-3.3$ & $<0.0001$ \\
\hline
\end{tabular}

* Only those with a p 0.05 are reported here

* This information was not available for all patients in study

baseline, there were no significant differences in the numbers of male and female subjects or in their mean ages (Table 3). However, there were significant differences in spirometry severity score, FEV1/FVC ratio, and FEF $25-75 \%$ between the males and females in this study.

\section{Outcome Measures}

The demographic information collected for each subject included age, sex, asthma severity as defined by NHLBI criteria (27), allergy skin test results, the diagnosis of allergic rhinitis, and LTM use. Assessment of asthma control was done pre and post introduction of LTM therapy. The measures recorded for each patient included frequency of nocturnal awakenings, exercise tolerance, school absenteeism, provider's assessment of asthma control, rescue use of short acting bronchodilators, number of hospitalizations or sick visits, and spirometry severity. A score was assigned for each of these parameters as shown in Table 2. Additional spirometry data obtained for each subject included FEV1, FEV1 to forced vital capacity (FEV1/FVC) ratio, peak expiratory flow (PEF), and forced expiratory flow measured between $25 \%$ and $75 \%$ of the vital capacity (FEF 25-75\%). Documented reference standards (28) were used to determine baseline lung functions. Pulmonary function tests were standardized and performed by the same technician for all subjects, and results were recorded as percentages (actual/ reference x 100).

The dose and type of ICS used by each subject were also noted before treatment with a LTM and at the follow-up appointment after initiating treatment. Each dose was standardized by conversion to beclomethasone dipropionate (BDP) equivalence units (mcg) as described (29) Finally, it was also noted if and why LTM therapy was discontinued.

\section{Statistical Analysis}

All statistical analyses were performed using a computer program (StatView). The efficacy of LTMs was assessed by comparing changes in pre and post outcome measures in the form of scores, percentages, and BDP dose. For the analyses of spirometry data, each percent change was tabulated as the change in percent predicted values. Statistical comparisons were performed using paired t-tests for the pre and post comparisons, and unpaired t-tests with Bonferonni corrections for the specific LTM comparisons (given as $\mathrm{p}<0.05 / \mathrm{n}$, where $\mathrm{n}$ is the number of comparisons). 
Table 4. Before and After Assessment Scores and Pulmonary Function Test Results* with Standard Deviations for Each

\begin{tabular}{|c|c|c|c|c|}
\hline Variable & Pre & Post & Change & Level of Significance \\
\hline Frequency of nocturnal awakenings & $1.0+/-0.11$ & $0.7+/-0.11$ & -0.3 & 0.07 \\
\hline Exercise tolerance & $0.5+/-0.07$ & $0.4+/-0.06$ & -0.1 & 0.26 \\
\hline School absenteeism & $0.9+/-0.10$ & $0.7+/-0.10$ & -0.2 & 0.45 \\
\hline $\begin{array}{l}\text { Provider's assessment of asthma } \\
\text { control }\end{array}$ & $1.0+/-0.10$ & $0.6+/-0.09$ & -0.4 & 0.0005 \\
\hline $\begin{array}{l}\text { Rescue use of short acting } \\
\text { bronchodilators }\end{array}$ & $1.0+/-0.12$ & $0.7+/-0.11$ & -0.3 & 0.07 \\
\hline $\begin{array}{l}\text { Number of hospitalizations or } \\
\text { sick visits }\end{array}$ & $0.9+/-0.14$ & $0.2+/-0.06$ & -0.7 & $<0.0001$ \\
\hline Inhaled corticosteroid dose+ & $1683.5+/-136.5$ & $1636.2+/-130.0$ & -47.3 & 0.72 \\
\hline Use of oral steroids in past six months & $0.5+/-0.05$ & $0.3+/-0.05$ & -0.2 & 0.0015 \\
\hline Spirometry severity & $1.3+/-0.12$ & $1.0+/-0.12$ & -0.3 & 0.0015 \\
\hline FEV1 & $82.0+/-2.05$ & $87.8+/-2.01$ & +5.7 & 0.0002 \\
\hline FEV1/FVC & $78.0+/-1.19$ & $81.5+/-1.28$ & +3.5 & 0.0033 \\
\hline $\mathrm{PEF}$ & $91.3+/-2.39$ & $98.6+/-2.61$ & +7.3 & 0.0039 \\
\hline FEF $(25-75 \%)$ & $67.8+/-3.36$ & $76.0+/-3.20$ & +8.2 & 0.0042 \\
\hline
\end{tabular}

*Based on the \% reference according to each individual patient's age, sex, and height.

+This parameter is not a score but the actual dose converted into beclomethasone units (mcg)

d Statistically significant p-values accounting for non-independence

Measurements are expressed as means standard error of the mean, and a $\mathrm{p}$ value of $£ 0.05$ was considered significant.

\section{RESULTS}

Overall treatment comparison. Statistically significant treatment effects of montelukast and zafirlukast were noted in 8 of the 13 parameters studied. Notably, there were significant improvements in providers' assessment of asthma control and in the number of hospitalizations or sick visits (Table 4). There were also significant improvements in all of the spirometry parameters measured, including the spirometry severity score (Table 4).

Furthermore, LTM therapy resulted in a statistically significant decline in the use of oral steroids during the past six months. About half $(50.6 \%)$ of the patients in this study had severe persistent asthma, and naturally more of these patients $(66.6 \%)$ utilized oral steroids than patients with moderate $(37.5 \%)$ or mild $(22.2 \%)$ persistent asthma. After LTM treatment, only $35.7 \%$ of patients with severe persistent asthma, for example, were on oral steroids, indicating that almost half of this group of patients was tapered off of oral steroids within six months. In contrast, there appeared to be no effect of LTM treatment on ICS dosage overall, such that twenty patients had their dosage increased, 21 patients had their dosage decreased, and 42 patients remained on the same dosage.

Subgroup analysis I: Comparison of continued and discontinued group. Fifteen patients (18.1\%) discontinued their LTM treatment but were included in all of the analyses as an intention-to-treat. The reasons for discontinuing LTMs were varied, with the two most important being lack of effectiveness $(n=7)$ and side effects such as headaches $(n=3)$. Other reported reasons for discontinuing were difficulty of administration $(n=2$; disliked the taste and disliked swallowing the pills), switching from one LTM to the other $(\mathrm{n}=2$; switched from montelukast to zafirlukast and vice-versa), or problems with compliance $(n=1)$. There were no statistically significant differences between the continued $(\mathrm{n}=68 ; 81.9 \%)$ and discontinued groups in age, asthma severity, presence of skin allergies 
Table 5. Patient Comparison in Relation to Specific LTM Used (Pretreatment)

\begin{tabular}{lll}
\hline & Montelukast & Zafirlukast \\
\hline $\begin{array}{l}\text { Age (yrs) (range 5- } \\
16 \text { yrs) }(\mathrm{p}=0.0235)\end{array}$ & $10.4+/-0.24$ & $11.9+/-0.51$ \\
$\begin{array}{l}\text { Beclomethasone } \\
\text { dose of ICS } \\
(\mathrm{p}=0.0001)\end{array}$ & $1479+/-91.9$ & $2841+/ 266.6$ \\
$\begin{array}{l}\text { Number of patients } \\
\text { Mild asthma ( } \mathrm{n}=9)\end{array}$ & $92(86.7 \%)$ & $11(13.3 \%)$ \\
$\begin{array}{l}\text { Moderate asthma } \\
\text { (n=32) }\end{array}$ & 26 & 0 \\
$\begin{array}{l}\text { Severe asthma } \\
(\mathrm{n}=42)\end{array}$ & 37 & 6 \\
$\begin{array}{l}\text { Number of points } \\
\text { that discontinued } \\
\text { prescribed LTM }\end{array}$ & 8 & 7 \\
\hline
\end{tabular}

and/ or allergic rhinitis, or any pre and post outcome parameter (data not shown).

Subgroup analysis II: Comparing montelukast and zafirlukast. The characteristics of subjects prescribed montelukast versus zafirlukast differed in only a few parameters as shown in Table 5 . Patients who used montelukast used statistically significant lower dosages of ICS at the start of treatment compared with zafirlukast users, although there was no overall change in ICS dose after initiation of LTMs in either group. However, there were no statistically significant differences between the two groups in terms of night symptoms, exercise tolerance, school absenteeism, providers' assessment, use of short acting bronchodilators, number of hospitalizations or sick visits, spirometry severity score, or any spirometry measurements (data not shown). It is unknown why zafirlukast was not prescribed for any patient with mild persistent asthma.

\section{DISCUSSION}

Leukotriene antagonists represent a new class of asthma therapy, and it is yet unclear how best to utilize LTMs. This study evaluated outcomes that might be expected with the integration of LTMs into the management of children with persistent asthma.

Main Findings

The results from the present study indicate that the greatest benefit in terms of asthma improvement to be gained from LTM use is in pulmonary function. All spirometry parameters increased following the use of montelukast or zafirlukast. Moreover, significant treatment effects were noted in four other parameters: physician assessment, number of hospitalizations and sick visits (both emergency department and clinic visits), use of oral steroids, and spirometry severity score. LTMs may thus help to reduce the asthma hospitalization rate among children 14 years of age living in Hartford, which is currently much higher (at 57.1 per 10,000$)$ than the rates in the entire state and in the US-37 (30). Likewise, utilization of LTMs has the potential to help reduce the rate of annual asthma emergency room visits among children 14 years of age, which are currently higher in Hartford (at 256 per $10,000)$ than anywhere else in the state $(30)$.

The frequency of nocturnal awakenings did not decline significantly, a result consistent with previous studies using both zafirlukast19 and montelukast.5 However, other symptom scores, namely 2 agonist requirement, days missed from school and ICS dose, were not affected by LTM treatment, contradicting previous studies $(31,32)$. For example, one study found that LTM use allowed for an existing ICS dose to be halved without deterioration in symptoms or lung function (32). This result had provided the impetus for initiating LTM treatment in some of the children in this study who had experienced growth and/or height retardation due to ICS use. The present study, however, found no overall change in ICS dose most likely because an equal number of patients had increases or decreases in their dosage during addition of LTMs to their existing treatment.

The 1997 NHLBI asthma treatment guidelines specify the use of LTMs only as alternatives to lowdose inhaled corticosteroids, disodium cromoglycate, nedocromil, or sustained-release theophylline in patients with mild persistent asthma (2). However, since then, studies have shown that LTMs are efficacious over a wider range of asthma severity. One study found that zafirlukast given to patients $>12$ years with continuing asthma symptoms despite a high dose of ICS resulted in a significant reduction in the risk of worsening asthma symptoms as compared to placebo (33). Similarly, it has been reported that montelukast may confer additional improvement of asthma control when used concurrently with ICS in moderate to severe asthma (31). The present study supports these findings since addition of LTMs to the treatment regimen of patients with moderate to severe asthma and already on ICS (with the exception of two subjects) led to a significant improvement in a majority of assessment parameters. This finding points to the importance of LTMs in helping to control mediators of asthma inflammation (i.e. leukotrienes) that cannot be controlled with corticosteroids alone. 


\section{Additional Findings}

The data for the continued and discontinued groups are similar to those found in other studies. The Mediplus database, which includes the prescription records of 123 patients on montelukast, indicate that less than $25 \%$ of these patients discontinued the drug.34 Unfortunately, the exact reasons for discontinuing in that study are not available, although it is speculated that the reasons may include lack of effectiveness and/or adverse effects. The same reasons for cessation were found in the present study, with lack of effectiveness as the reason for 7 of 15 patients.

A comparison of montelukast and zafirlukast use in this study implies that these two LTMs have similar efficacy and tolerability. Since previous studies of LTMs have focused on only a single LTM, such comparisons are lacking in the literature. It has been proposed that montelukast, with its lack of dosing considerations and drug interactions (35) may be better tolerated than zafirlukast.

\section{Characteristics and Limitations}

Baseline measures indicate that the patient population examined in this study is not characteristic of all asthma patients. First, the number of children with asthma in Hartford is significantly higher than the national average. Second, as reported in other studies (36), there is a referral bias in this population such that the children seen by the pediatric pulmonary practice are more severely asthmatic. In this study, $51 \%$ of the patients had severe asthma while only $10 \%$ had mild asthma. This trend is nearly opposite of estimates of national asthma rates in which $60 \%$ of children have mild asthma, $30 \%$ have moderate asthma, and $10 \%$ have severe asthma (37). A population of children more equal with regards to the type of persistent asthma would perhaps be a more ideal population to have studied. However, it is already recommended that LTMs could or should be considered as alternative long term controllers in patients with mild persistent asthma (2). Moreover, it has been suggested that patients at all levels of asthma severity and requiring high doses of inhaled corticosteroids should be given a trial of LTMs (38). Thus, this study sample is valuable given that the full therapeutic potential range of LTMs is as yet unconfirmed.

Another baseline characteristic worth mentioning is the statistically different spirometry results for males and females. The reason why males performed worse on lung function tests overall is unknown but may be related to the higher degree of bronchial lability (39) and higher occurrence of respiratory infections among males compared to females (37). Another reason may be that asthma is generally worse in boys than in girls. It has also been suggested that pulmonary function differs between male and female children due to gender differences in both mechanical properties of the lung and the inflammatory process (40).

Improvements seen in this cohort of patients may not be solely due to addition of LTMs to their treatment regimens. Confounding variables include concomitant variations in all other medications taken by a patient during therapy with a LTM. For example, twenty of the patients in this study had their ICS dose increased at the same time as a LTM was added to their list of daily medications. It is thus not possible to accurately know how much the observed improvements were due to the increased ICS doses. Nonetheless, these results suggest that montelukast and zafirlukast are effective in treating persistent asthma when used concomitantly with an ICS. Another limitation is selection bias since the characteristics of the 30 patients excluded from the study are unknown and may have influenced the overall results of this study.

\section{Future Studies}

In summary, the results from this study thus indicate that CysLT1 selective receptor antagonists are clinically effective in the treatment of children with persistent asthma. This conclusion is most applicable to montelukast given the relatively small number of patients in the zafirlukast group. Additional efficacy data from long-term studies are required to establish the position of montelukast and zafirlukast in asthma treatment guidelines. More research will also be needed to assess the long-term efficacy and safety of LTMs.

\section{ACKNOWLEDGEMENTS}

I thank Dr. K. Daigle for guidance during this project and for comments on earlier versions of this manuscript, Dr. C. Schramm for help with statistical analysis and for comments and discussion, and Dr. P. Kehoe for providing the opportunity to perform this

\section{REFERENCES}

1. Adams PF, Marano MA. Current estimates from the National Health Interview Survey, 1994. Vital Health Stat 10:94; 1995.

2. National Asthma Education and Prevention Program. Highlights of the Expert Panel Report II: Guidelines for the Diagnosis and Management of Asthma. Bethesda, MD: National Institutes of Health. Publication No. 97-4051A; 1997.

3. Allen DB. Do inhaled steroids impair growth? It all depends. J Respir Dis 19:1023-1036; 1998. 
4. Lemanske RF, Allen DB. Choosing a long-term controller medication in childhood asthma. Am J Respir Crit Care Med 156:685-687; 1997.

5. Knorr B, Matz J, Bernstein JA, et al. Montelukast for chronic asthma in 6- to 14- year-old children: a randomized, double-blind trial. Pediatric Montelukast Study Group. JAMA 279:1181-1186; 1998.

6. Fraser CM, Nelson HS, Middleton E Jr. Adrenergic agents. In: Middleton E Jr, Reed CE, Ellis EF, et al, editors. Allergy: Principles and Practice. St Louis, MO: Mosby-Year Book, 1993.

7. Powell EC, Reynolds SL, Rubenstein JS. Theophylline toxicity in children: a retrospective review. Pediatr Emerg Care 9(3):129-33; 1993.

8. Drazen JM, Israel E, O'Byrne PM. Treatment of asthma with drugs modifying the leukotriene pathway. N Engl J Med 340:197-206; 1999.

9. Korenblat PE. The role of antileukotrienes in the treatment of asthma. Ann Allergy Asthma Immunol 86(Suppl):31-39; 2001.

10. Weisberg SC. Pharmacology of asthma in children, with special reference to leukotriene receptor antagonists. Pediatr Pulmonol 29:46-61; 2000.

11. Accolate. Manufacturer's prescription information. Zeneca Pharmaceuticals: Carolina, Puerto Rico; 2001.

12. Zyflo Filmtab. Manufacturer's prescription information. Abbott Laboratories: Chicago, IL, USA; 2000.

13. Singulair. Manufacturer's prescription information. Merck \& Co, Inc: Whitehouse Station, NJ, USA; 2001.

14. Rachelefsky G, Bruton S, Barnes PJ, et al. Airway remodeling: The inflammatory response in asthma therapy. Dialogues in Asthma Management 1:1-9; 1998.

15. Evans JF. Cysteinyl leukotriene receptors. Prostaglandins Other Lipid Mediat 68-69:587- 597; 2002.

16. Adkins JC, Brogden RN. Zafirlukast. A review of its pharmacology and therapeutic potential in the management of asthma. Drugs 55:121-144; 1998.

17. McGill KA, Busse WW. Zileuton. Lancet 348:519-524; 1996.

18. Lazarus SC, Lee T, Kemp JP, et al. Safety and clinical efficacy of zileuton in patients with chronic asthma. Am J Manag Care 4:841-848; 1998.

19. Spector SL, Smith LJ, Glass M. Effects of 6 weeks of therapy with oral doses of ICI 204,219, a leukotriene D4 receptor antagonist, in subjects with bronchial asthma. ACCOLATE Trialists Group. Am J Respir Crit Care Med 150:618-623; 1994.

20. Lampl KL, Dixon SJ, Bonuccelli CM. Long-term safety and efficacy of zafirlukast (Accolate) in pediatric patients with mild-to-moderate asthma. Am J Respir Crit Care Med 159(3):S138; 1999.

21. Bisgaard H, Nielsen KG. Bronchoprotection with a leukotriene receptor antagonist in asthmatic preschool children. Am J Respir Crit Care Med 162:187-190; 2000.

22. Kemp JP, Dockhorn RJ, Shapiro GG, et al. Montelukast once daily inhibits exercise- induced bronchoconstriction in 6-14year old children with asthma. J Pediatr 133(3):424-8; 1998.

23. Pearlman DS, Lampl KL, Dowling PJ Jr., Miller CJ, Bonuccelli CM. Effectiveness and tolerability of zafirlukast for the treatment of asthma in children. Clin Ther 22(6):73247; 2000.

24. Kemp JP. Guidelines update: where do the new therapies fit in the management of asthma? Drugs 59 Suppl 1:23-28; discussion 43-5; 2000.

25. Strek ME. Consensus guidelines for asthma therapy. Ann Allergy Asthma Immunol 86(Suppl):40-44; 2001.

26. Katial RK, Stelzle RC, Bonner MW, Marino M, Cantilena LR, Smith LJ. A drug interaction between zafirlukast and theophylline. Arch Intern Med 158(15):1713-5; 1998.

27. National Asthma Education and Prevention Program. Highlights of the Expert Panel Report II: Guidelines for the Diagnosis and Management of Asthma. Bethesda, MD: National Institutes of Health. Publication No. 97-4051A; 1997. Figure 1-3.

28. Taussig LM, Chernick V, Wood R, Farrell P, Mellins RB. Standardization of lung function in testing in children. Proceedings and recommendations of the GAP conference committee, Cystic Fibrosis Foundation. J Pediatrics 97:668$676 ; 1980$.

29. National Asthma Education and Prevention Program. Highlights of the Expert Panel Report II: Guidelines for the Diagnosis and Management of Asthma. Bethesda, MD: National Institutes of Health. Publication No. 97-4051A; 1997. Tables 3-5b and 3-5c.

30. Connecticut Department of Public Health: Asthma in Connecticut. Hartford, CT; 2001.

31. Lipworth BJ: Systemic adverse effects of inhaled corticosteroid therapy. A systemic review and meta-analysis. Arch Intern Med 159:941-955; 1999.

32. Tamioki J, Kondo M, Sakai N, et al. Leukotriene antagonist prevents exacerbation of asthma during reduction of highdose inhaled corticosteroids. Am J Respir Crit Care Med 155:1235-1240; 1997.

33. Tashkin DP, Nathan RA, Howland WC, et al. An evaluation of zafirlukast in the treatment of asthma with exploratory subset analyses. J Allergy Clin Immunol 103 (2 Pt 1):246254; 1998.

34. Price D. Tolerability of montelukast. Drugs 59 Suppl 1:3542; 2000 .

35. Storms W, Michele TM, Knorr B, et al. Clinical safety and tolerability of montelukast, a leukotriene receptor antagonist, in controlled clinical trials in patients aged $>$ or $=6$ years. Clin Exp Allergy 31(1):77-87; 2001.

36. Ruurd JR, Gerritsen J, Van Aalderen WMC, et al. Risk factors for the persistence of respiratory symptoms in childhood asthma. Am Rev Respir Dis 148:1490-1495; 1993.

37. Canny GJ, Levison H. Asthma. In: Loughlin G, Levison H, editors. Respiratory Diseases in Children: Diagnosis and Management. Baltimore, MD: Williams and Wilkins, 1994.

38. Wenzel SE. Antileukotriene drugs in the management of asthma. JAMA 280:2068-69; 1998.

39. Verity CM, Ven Heule B, Carlswell F, Hughes AO. Bronchial lability and skin reactivity in siblings of asthmatic children. Arch Dis Child 42:542-548; 1984.

40. Gold DR, Wypij D, Wang X, et al. Gender- and race-specific effects of asthma and wheeze on level and growth of lung function in children in six U.S. cities. Am J Respir Crit Care Med 149:1198-1208; 1994.

Duarte Machado is a rising third year medical student at the University of Connecticut School of Medicine, USA. He holds a B.S. in Neuroscience from Trinity College, Hartford, Connecticut, USA. His research interests include understanding the pathophysiology of asthma and its management and treatment. He is planning a career in internal medicine. 